\title{
Efeitos do Uso de Tecnologias da Informaçấo e Comunicação na Capacitaçáo de Cuidadores de Crianças com Autismo ${ }^{1}$ EFFECTS OF THE USE OF INFORMATION AND COMMUNICATION TECHNOLOGIES ON the TRAINING of CAREGIVERS of CHILDREN WITH AUtisM
}

\author{
Camila Graciella Santos GOMES² \\ Analice Dutra SILVEIRA ${ }^{3}$ \\ Letícia Pedroso Castelo Branco ESTRELA ${ }^{4}$ \\ Ana Luíza Barbosa FIGUEIREDO ${ }^{5}$ \\ Amanda Queiroz de OLIVEIRA ${ }^{6}$ \\ Ianaiara Marprates OLIVEIRA ${ }^{7}$
}

\begin{abstract}
RESUMO: O objetivo geral deste estudo foi avaliar a viabilidade do uso de Tecnologias da Informação e Comunicação para a capacitação de cuidadores de crianças com autismo em um contexto de Intervenção Comportamental Intensiva. Participaram do estudo 24 crianças com o diagnóstico de autismo, com idades entre 3 anos e 2 meses e 8 anos e 10 meses e seus respectivos cuidadores. Os cuidadores realizaram as estimulaçôes comportamentais e intensivas com as crianças com autismo e foram capacitados por profissionais especializados, porém essa capacitação ocorreu por meio do uso de Tecnologias da Informação e Comunicação, estando profissionais e cuidadores em locais diferentes. Os participantes com autismo foram avaliados antes e após a intervenção, por meio de instrumentos padronizados que permitiram medir o desenvolvimento das crianças. Os resultados indicaram a viabilidade do uso dessas tecnologias para capacitar cuidadores e ganhos no desenvolvimento das crianças com autismo.
\end{abstract}

PALAVRAS-CHAVE: Transtornos do Espectro do Autismo. Educação Especial. Cuidadores. Análise do Comportamento Aplicada. Tecnologias da Informação e Comunicação.

\begin{abstract}
The objective of this study was to evaluate the viability of using Information and Communication Technologies for the training of caregivers of children with autism in the context of Intensive Behavioral Intervention. Twenty-four children diagnosed with autism, aged between 3 years and 2 months and 8 years and 10 months and their respective caregivers participated in the study. Caregivers performed behavioral and intensive stimulations with children with autism and were trained by specialized professionals, however this training took place using Information and Communication Technologies, being professionals and caregivers in different locations. Participants with autism were assessed before and after the intervention, using standardized instruments that allowed measuring the children's development. The results indicated the viability of using these technologies to empower caregivers and gains in the development of children with autism.
\end{abstract}

KEYWORDS: Autism Spectrum Disorders. Special Education. Caregivers. Applied Behavior Analysis. Information and Communication Technologies.

\footnotetext{
${ }^{1}$ https://doi.org/10.1590/1980-54702021v27e0085

${ }^{2}$ Doutora em Educação Especial pela Universidade Federal de São Carlos (UFSCar). Diretora do Centro de Estudos e Intervenção para o Desenvolvimento Humano (CEI Desenvolvimento Humano). Docente da Faculdade de Ciências Médicas de Minas Gerais e membro do Instituto Nacional de Ciência e Tecnologia sobre Comportamento, Cognição e Ensino (FAPESP: 2014/50909-8; CNPq 465686/2014-1; CAPES: 88887.136407/2017-00). Belo Horizonte/Minas Gerais/Brasil. E-mail: camila.gomes@ceidesenvolvimentohumano.com.br. ORCID: http://orcid.org/0000-0002-4268-328X

${ }^{3}$ Mestra em Educaçấo Especial pela UFSCar e diretora do CEI Desenvolvimento Humano. Belo Horizonte/Minas Gerais/Brasil. E-mail: analice.silveira@ceidesenvolvimentohumano.com.br. ORCID: http://orcid.org/0000-0002-2299-4443

${ }^{4}$ Graduanda em Psicologia pela Faculdade de Ciências Médicas de Minas Gerais. Belo Horizonte/Minas Gerais/Brasil. E-mail: leticiapcestrela@gmail.com. ORCID: https://orcid.org/0000-0002-9517-0314

${ }^{5}$ Graduanda em Psicologia pela Faculdade de Ciências Médicas de Minas Gerais. Belo Horizonte/Minas Gerais/Brasil. E-mail: analuizabf97@gmail.com. ORCID: https://orcid.org/0000-0001-5641-5959

${ }^{6}$ Graduanda em Psicologia pela Faculdade de Ciências Médicas de Minas Gerais. Belo Horizonte/Minas Gerais/Brasil. E-mail: amandaqoliv@gmail.com. ORCID: https://orcid.org/0000-0002-3740-6974

${ }^{7}$ Psicóloga do CEI Desenvolvimento Humano. Belo Horizonte/Minas Gerais/Brasil. E-mail: ianaiaraprates@gmail.com. ORCID: http://orcid.org/0000-0001-5603-2173
} 


\section{INTRODUÇÁo}

O autismo (Transtornos do Espectro do Autismo [TEA]) é um transtorno do neurodesenvolvimento caracterizado por alterações na comunicação social e pela presença de interesses restritos, comportamentos repetitivos e estereotipados (American Psychiatry Association [APA], 2013). Atualmente, não há cura para o transtorno, porém há descriçóes na literatura a respeito de diversas intervençóes terapêuticas que podem promover ganhos no desenvolvimento das pessoas afetadas e diminuição dos sintomas, resultando em melhora da qualidade de vida, independência e inserção social (Medavarapu et al., 2019).

Dentre as intervençóes terapêuticas com maior evidência de efetividade estáo os modelos de "Intervenção Comportamental Intensiva", conhecidos no Brasil como "Terapia ABA" (Medavarapu et al., 2019; Romanczyk \& McEachin, 2016), caracterizados por estimulações que ocorrem predominantemente de maneira individualizada (um educador para uma criança com autismo), realizadas por muitas horas semanais (de 15 a 40 horas), por pelo menos dois anos consecutivos, que abrangem várias áreas do desenvolvimento simultaneamente e que são fundamentadas em princípios de Análise do Comportamento (Baer et al., 1987).

O primeiro estudo sobre Intervenção Comportamental Intensiva aplicada ao tratamento do autismo foi publicado na década de 1980 (Lovaas, 1987) e, após essa publicação, dezenas de estudos posteriores replicaram parcialmente os resultados do estudo inicial, utilizando educadores diferentes (estudantes, profissionais e familiares) e contextos múltiplos (instituiçōes especializadas, escolas e a residência das crianças), e indicaram, no geral, melhora no desenvolvimento dos participantes com autismo após esse tipo de intervenção, especialmente quando ela é realizada precocemente (Medavarapu et al., 2019; Smith et al., 2000; Warren et al., 2011).

A oferta de modelos de Intervenção Comportamental Intensiva para o tratamento do autismo no Brasil ainda é escassa, em função da demanda, que é maior do que o número de profissionais disponíveis para fazer esse tipo de intervenção. Até o momento não há estudos amplos a respeito da prevalência do autismo no país, porém, se considerarmos a prevalência de um caso de Transtorno do Espectro do Autismo a cada 59 pessoas, indicada por Baio et al. (2018), podemos projetar que, na populaçáo brasileira, estimada em aproximadamente 210.000.000 habitantes (Instituto Brasileiro de Geografia e Estatística [IBGE], 2019), teríamos aproximadamente 3.500.000 pessoas com autismo. Segundo o Conselho Federal de Psicologia, em 2019, havia aproximadamente 340.000 psicólogos no Brasil (Conselho Federal de Psicologia [CFP], 2019); isso significa que mesmo que todos os psicólogos se dedicassem exclusivamente a acolher a essa demanda relativa ao autismo, cada psicólogo brasileiro teria de atender a aproximadamente 10 pessoas com esse diagnóstico; são, assim, números difíceis de serem alcançados. Outra questão importante é que intervençôes comportamentais são tipicamente planejadas por analistas do comportamento e, se considerarmos especificamente a quantidade de analistas do comportamento no Brasil, teremos um número bem inferior de profissionais. A Associação Brasileira de Psicoterapia e Medicina Comportamental indicava aproximadamente 100 analistas do comportamento acreditados no ano de 2019, o que significa que cada profissional teria de atender a aproximadamente 35.000 pessoas com autismo (Associação Brasileira de Psicologia e Medicina Comportamental [ABPMC], 2019). 
Dificuldades relacionadas ao acesso a Intervenções Comportamentais Intensivas, historicamente, não ocorrem apenas no Brasil. Smith et al. (2000) ressaltam que a escassez de profissionais e o alto custo financeiro desse tipo de intervenção, em função das muitas horas semanais de terapia necessárias, dificultam a obtenção do tratamento. Os pesquisadores enfatizam que se tornou inviável utilizar profissionais especializados para realizar todo o tratamento e que a solução mais comum para implementar esse tipo de intervenção tem sido por meio do emprego de cuidadores (pais, familiares, babás, estagiários e entre outros) para a realização das estimulaçôes, com orientação e supervisão de terapeutas comportamentais capacitados (Anderson et al., 1987; Dawson et al., 2010; McConachie \& Diggle, 2007; Sheinkopf \& Sielgel, 1998; Sherman et al., 1988; Smith et al., 2000).

Diante desse contexto, torna-se necessário pensar em alternativas para disponibilizar Intervençóes Comportamentais Intensivas a um número maior de pessoas com autismo no Brasil. Estudos nacionais descreveram efeitos importantes desse tipo de intervenção com crianças brasileiras e indicaram a viabilidade da capacitação dos cuidadores para a realização das estimulaçóes necessárias ao tratamento, que seguiram as orientações de terapeutas comportamentais capacitados (Andalécio et al., 2019; Gomes et al., 2017; Gomes et al., 2019). Nesse sentido, as Tecnologias da Informação e Comunicação (TICs) podem ser instrumentos úteis para atender à demanda de pessoas com autismo no Brasil. O uso de plataformas para videoconferência e aplicativos de mensagens, por exemplo, podem ser recursos valiosos para propiciar acesso a intervençóes especializadas por pessoas que não estão fisicamente próximas a profissionais capacitados.

Assim sendo, o objetivo geral deste estudo foi avaliar a viabilidade do uso de TICs para capacitar cuidadores de crianças com autismo em um contexto de Intervenção Comportamental Intensiva. Isso significa que os cuidadores realizaram as estimulaçóes comportamentais e intensivas com as crianças com autismo e foram orientados por profissionais especializados, porém essa orientação ocorreu por meio do uso de TICs, estando profissionais e cuidadores em locais diferentes, sem contato direto (presencial).

Alguns estudos investigaram a utilização de TICs para o tratamento do autismo. Boisvert et al. (2010), em um estudo de revisão sistemática, analisaram oito pesquisas que envolveram o uso de videoconferência para ensinar profissionais ou pais a fazer intervençóes variadas. No Brasil, alguns estudos também usaram TICs para ensinar pais de pessoas com autismo a fazer intervençôes pontuais (Barboza et al., 2015; Barboza et al., 2019). Entretanto, até o momento, nenhum estudo investigou o uso de TICs para a capacitação de cuidadores de crianças com autismo em um contexto de Intervenção Comportamental Intensiva. $\mathrm{O}$ estudo que mais se aproximou dessa temática foi o de Vismara et al. (2009), que capacitou profissionais por meio de videoconferência para a realização de um modelo de Intervenção Comportamental Intensiva.

O estudo atual é inédito por investigar o uso de TICs para a capacitação de cuidadores de crianças com autismo em um contexto de Intervenção Comportamental Intensiva (quando se realiza de 15 a 40 horas semanais de estimulaçáo). Os resultados podem ser importantes não só para ampliar a compreensão a respeito de intervençóes efetivas na área do autismo, no campo da Educação Especial, como também indicar alternativas para oportunizar 
intervenções de qualidade a pessoas com esse diagnóstico que não têm acesso presencial a profissionais capacitados.

\section{Método}

Nesta seção, discorremos sobre os participantes da pesquisa, os instrumentos de avaliação utilizados, as TICs que foram usadas como recursos e os procedimentos de coletada de dados. Além disso, abordamos as consideraçóes éticas e de fidedignidade levadas em conta nesta pesquisa.

\subsection{Participantes}

Participaram do estudo 24 crianças com autismo e seus respectivos cuidadores, que foram atendidos pelo Centro de Estudos e Intervenção (CEI) para o Desenvolvimento Humano entre 2013 e 2019. É importante ressaltarmos que o cuidador de cada criança era designado pela família dela e nem sempre era o responsável legal por ela. Todas as crianças tinham o diagnóstico prévio de autismo realizado por psiquiatra ou neurologista infantil especializados, independentes do CEI, seguindo critérios da CID-10 (Organização Mundial da Saúde [OMS], 1993), do DSM-IV-TR (APA, 2002) ou do DSM-V (APA, 2013). O critério escolhido dependia da preferência do médico que realizou o diagnóstico, sem nenhuma influência do CEI.

Os participantes com autismo, além de realizarem Intervenção Comportamental Intensiva no CEI, faziam sessóes individuais de fonoaudiologia e/ou de terapia ocupacional em outras instituiçóes independentes do CEI, uma a duas vezes por semana em consultório (não eram intervenções domiciliares e nem focadas na capacitação dos cuidadores), com duração média de 40 minutos cada sessão. Todas as crianças frequentavam escolas regulares, por aproximadamente 20 horas semanais.

\subsection{InSTRUMENTOS DE AVALIAÇÁO}

Foram utilizados o Inventário Portage Operacionalizado - IPO (Williams \& Aiello, 2001), o Psychoeducational Profile-Revised-PEP-R (Schopler et al., 1990) e a Childhood Autism Rating Scale - CARS (Schopler et al., 1988) para avaliar os participantes com autismo.

O IPO, adaptado e operacionalizado para a população brasileira por Williams e Aiello (2001), mede o padrão de desenvolvimento infantil em cinco áreas: linguagem, socialização, desenvolvimento motor, cognição e autocuidados, em períodos de idade que vão de 0 a 6 anos. Destina-se a avaliar o desenvolvimento de qualquer criança, independentemente de algum tipo de diagnóstico.

O PEP-R avalia tanto atraso no desenvolvimento como comportamentos típicos de autismo e oferece informações sobre sete áreas na Escala de Desenvolvimento: imitação, percepção, coordenação motora fina, coordenação motora grossa, integração olho mão, desenvolvimento cognitivo e cognitivo verbal, além de quatro áreas na Escala de Comportamento: linguagem, relacionamento e afeto, respostas sensoriais e interesses por materiais. Pode ser utilizado com crianças de 6 meses a 12 anos de idade. Foi adaptado e validado para a popu- 
lação brasileira por Leon et al. (2004). Neste estudo, foram utilizados os dados da Escala de Desenvolvimento (não os da Escala de Comportamento).

A CARS permite identificar crianças com características comportamentais de autismo. De acordo com a escala, os resultados da avaliação podem ser distribuídos em três categorias: desenvolvimento normal (15-29,5), autismo leve/moderado (30-36,5) e autismo grave (acima de 36,5). Pode ser utilizada com crianças acima de 36 meses de idade. Essa escala foi adaptada e validada para a população brasileira por Pereira et al. (2008). É importante esclarecer que a CARS não é um instrumento de diagnóstico de autismo; ela categoriza a criança em função da quantidade e da severidade dos sintomas. Dessa maneira, "desenvolvimento normal" não significa que a criança não tenha autismo, mas indica que a criança tem poucos sintomas de autismo.

Os participantes com autismo que tinham idades acima de 5 anos não foram avaliados pelo IPO, pois esse instrumento não é adequado para avaliar crianças acima de 6 anos (não seria possível realizar uma segunda avaliação). Todos os participantes foram avaliados pelo PEP-R e pela CARS.

\subsection{Tecnologias da InformaÇáo e ComunicaÇáo}

Dois recursos foram utilizados para a realização da capacitação dos cuidadores: 1) softwares ou aplicativos que permitiam conexóes de voz e vídeo para realização de videoconferência (teleatendimento); e 2) aplicativo de mensagens WhatsApp ${ }^{\circledR}$, que permitia a formação de grupos e o envio de mensagens de texto e voz, vídeos e imagens. Para as videoconferências, foram utilizados o Skype ${ }^{\oplus}$, Google Hangouts ${ }^{\oplus}$ e WhatsApp ${ }^{\oplus}$, de acordo com a preferência de cada cuidador.

\subsection{Procedimentos}

Os procedimentos utilizados nesta pesquisa foram: avaliação dos participantes com autismo, uso de TICs na capacitaçáo dos cuidadores e atividades realizadas pelos cuidadores.

\subsubsection{Avaliação dos Participantes COM Autismo}

Os efeitos da capacitação dos cuidadores, realizada com o uso de TICs, foram medidos por meio do desempenho das crianças com autismo nas avaliaçóes. Como os cuidadores realizaram as estimulaçóes comportamentais e intensivas com as crianças com autismo, a melhora no desempenho das crianças pode indicar que os cuidadores seguiram as orientaçóes dos profissionais especializados e foram efetivos em ensinar as crianças com autismo.

Inicialmente, as crianças foram avaliadas (A1: pré-testes) por dois profissionais do CEI, um com formação em Psicologia e o outro em Terapia Ocupacional. A aplicação dos instrumentos de avaliação foi realizada na sede do CEI (P2, P4, P8, P9, P10, P12, P14, P15, P17, P18, P19, P20, P23) ou na residência das crianças (P1, P3, P5, P6, P7, P11, P13, P16, P21, P22, P24). O PEP-R foi realizado em um ambiente com poucos estímulos, em situação na qual um terapeuta apresentava as atividades, e o outro registrava o desempenho da criança. O IPO foi aplicado por meio de entrevista realizada com os responsáveis pela criança com autismo. 
A CARS foi preenchida pelos profissionais com base na observação do comportamento da criança. Foram necessárias, para cada criança com autismo, em média, quatro horas para a aplicação dos instrumentos de avaliação, análise dos dados e redação de relatório com os resultados individuais. Em seguida, a intervenção era iniciada. Após 9 a 18 meses, as crianças foram avaliadas novamente pelos mesmos instrumentos utilizados na avaliação inicial (A2: pós-testes) em situações semelhantes à avaliação inicial. $\mathrm{O}$ tempo entre as avaliçóes dependeu da disponibilidade das famílias em viajar até a sede do CEI (no caso das avaliaçóes realizadas lá) ou da disponibilidade dos profissionais em ir até a residência dos participantes (quando a avaliação foi realizada na residência).

\subsubsection{Uso de TICs NA CAPACITAÇÁO DOS CUIDADORES}

Os cuidadores realizavam as atividades com suas respectivas crianças com autismo na residência dessas crianças. Para a realização das atividades, cada cuidador era instruído semanalmente por um profissional do CEI com formação em Psicologia, em sessóes de capacitação realizadas por videoconferência. A funçáo dos profissionais de Psicologia era ensinar os cuidadores a realizar as atividades com as crianças com autismo. Além disso, os profissionais também ensinavam os cuidadores a fazer registros sistemáticos do desempenho das crianças, em protocolos de papel, e supervisionavam a realização correta das atividades e dos registros, para garantir a qualidade e a fidedignidade da intervenção. Alguns participantes com autismo (P1, P2, P3, P4, P6, P7, P8, P9, P10, P11, P12, P13, P14, P15, P17, P18, P21, P23, P24) também eram acompanhados por um profissional de Terapia Ocupacional, que orientava os cuidadores quinzenalmente, por videoconferência, em relação à realização e ao registro de atividades específicas de autocuidados, que só foram inseridas com crianças que apresentavam desempenho baixo nessa área do desenvolvimento.

As sessóes realizadas pelos profissionais de Psicologia e de Terapia Ocupacional com os cuidadores, por meio de videoconferência, tinham dias e horários fixos. Pelo menos um dia antes de cada sessão, o cuidador deveria enviar as fotos dos protocolos preenchidos, referentes às atividades realizadas por ele com a criança durante a semana anterior, e diversos vídeos curtos (a quantidade de vídeos dependia da quantidade de atividades realizadas; cada vídeo tinha a duração média aproximada de dois minutos) com filmagens de trechos da aplicação dos procedimentos com a criança, para que os profissionais especializados pudessem acompanhar o desempenho da criança e do cuidador. Os protocolos e vídeos eram encaminhados por meio de um grupo criado em aplicativo de mensagens (WhatsApp ${ }^{\circledR}$ ) no qual estavam os profissionais especializados, os supervisores desses profissionais (responsáveis por auxiliar os profissionais na realização correta das orientações), o cuidador e os responsáveis pela criança. Os profissionais especializados só poderiam ver os vídeos e os protocolos em um computador de propriedade do CEI, por meio da versão do aplicativo de mensagens acessada em navegador (WhatsApp ${ }^{\circledR}$ Web), e nada poderia ser baixado em celulares pessoais. Todo o material enviado pelos cuidadores era arquivado em um disco rígido externo de propriedade do CEI, em pastas individuais para cada criança. 


\subsubsection{Atividades ReAlizadas Pelos Cuidadores}

Intervençóes Comportamentais Intensivas são caraterizadas pela implementação simultânea de muitas atividades, realizadas em 15 a 40 horas semanais, ao longo de meses de intervenção. Por isso, torna-se inviável a descrição, em um artigo científico, de todas as atividades realizadas (ver Lovaas, 1987), e a alternativa comumente utilizada nesse tipo de estudo é a publicação do conjunto de procedimentos utilizados em livros ou manuais, a fim de favorecer a replicabilidade dos procedimentos (ver Lovaas, 1981). Nesse sentido, os procedimentos utilizados nesse estudo foram publicados em três livros (Gomes, 2015; Gomes \& Silveira, 2016; Silveira \& Gomes, 2019).

Para cada criança foi estabelecido um currículo de habilidades básicas, conforme Gomes e Silveira (2016). Para as crianças acompanhadas por um profissional de terapia ocupacional, utilizou-se um currículo de habilidades de autocuidados, conforme Silveira e Gomes (2019). Para as crianças com demandas de ensino de leitura (P13, P17, P19, P20, P21), utilizou-se a rota de ensino descrita em Gomes (2015).

O currículo de habilidades básicas é dividido em cinco áreas: atenção, imitação, linguagem expressiva, linguagem receptiva e pré-acadêmica. Cada área apresenta programas de ensino em número variado, que se encontram detalhadamente descritos (Gomes \& Silveira, 2016), de modo a favorecer sua replicabilidade. O currículo de habilidades de autocuidados é dividido em quatro áreas: alimentação, higiene pessoal, vestuário e uso do banheiro, que englobavam habilidades variadas (ex. higiene pessoal: escovar os dentes, tomar banho, vestir e despir, entre outras), que também se encontram detalhadamente descritos (Silveira \& Gomes, 2019), de modo a favorecer sua replicabilidade. Os procedimentos de ensino de leitura utilizados foram os descritos por Gomes (2015).

O ensino das habilidades especificadas nos currículos era realizado pelos cuidadores, com suas respectivas crianças com autismo, seguindo as orientaçóes sistemáticas dos profissionais especializados. As orientações abordavam tanto as condiçóes de ensino: a preparação do ambiente e de materiais, como apresentar materiais e instruções, a exigência de resposta ativa da criança, a apresentação de consequências identificadas como potencialmente reforçadoras, os critérios para concluir cada tarefa ou cada programa, entre outros aspectos, como o manejo geral dos comportamentos da criança. Para cada programa, eram especificados critérios de aprendizagem, uso de reforçadores, procedimentos de correção e número de habilidades ensinadas por sessão.

Os cuidadores também registravam, em protocolos específicos para cada atividade, a data de realização da atividade, o número de tentativas de ensino e o desempenho da criança. Os protocolos utilizados eram simples (na maioria dos casos bastava marcar $\mathrm{x}$ ou $\mathrm{v}$ para acertos ou erros), para facilitar o registro dos cuidadores, conforme Gomes e Silveira (2016) e Silveira e Gomes (2019). Os procedimentos de ensino eram fundamentados em Análise do Comportamento, porém nenhuma capacitação teórica foi realizada com os cuidadores, apenas orientaçóes práticas e didáticas, com uso mínimo de termos técnicos.

As atividades eram realizadas pelos cuidadores, no geral, cinco vezes por semana, quatro horas por dia (três horas de atividades de habilidades básicas e/ou leitura e duas horas de autocuidados), totalizando em média de 15 a 25 horas semanais de estimulação para cada criança. Mais 
de um programa de ensino do currículo de habilidades básicas era inserido simultaneamente, dependendo da disponibilidade do cuidador e da criança. Em relação ao currículo de autocuidados, todas as habilidades eram ensinadas simultaneamente ao longo de todo o período de intervenção, sempre articuladas com as situaçóes de vida diária nas quais deveriam ocorrer.

\subsubsection{SÍNTESE DA SEQUÊNCIA DOS PROCEDIMENTOS}

A intervenção acontecia na seguinte sequência: 1) A criança era avaliada e o resultado da avaliação indicava quais currículos seriam utilizados com ela (por exemplo, se a criança não falava e era dependente nas atividades de autocuidados, os currículos de habilidades básicas e de autocuidados seriam implementados simultaneamente); 2) O supervisor, junto ao profissional responsável pelas sessões de capacitação do cuidador, selecionava quais, como e quando os programas de ensino seriam implementados, seguindo os critérios de ensino descritos nos currículos (Gomes, 2015; Gomes \& Silveira, 2016; Silveira \& Gomes, 2019); 3) Os protocolos das primeiras atividades a serem inseridas eram enviados por e-mail para um responsável pela criança; 4) O responsável pela criança deveria imprimir os protocolos e deixá-los à disposição para serem utilizados pelo cuidador na hora da videoconferência com o profissional; 5) A sessão realizada por videoconferência acontecia com o profissional e o cuidador da criança, sem a presença da criança; 6) O profissional explicava para o cuidador, de maneira didática, quais eram os objetivos de cada programa de ensino, como o cuidador deveria realizar as atividades com a criança e como ele deveria fazer os registros dessas atividades nos protocolos; 7) Ao término da sessão, o profissional fazia um resumo por escrito do que tinha sido orientado ao cuidador e enviava no grupo criado no aplicativo de mensagens, para que os responsáveis e o supervisor pudessem acompanhar as orientaçóes; 8) Ao longo da semana, o cuidador realizava com a criança as atividades que foram previamente combinadas na sessão com o profissional, registrava nos protocolos e fazia alguns vídeos breves nos quais deveriam aparecer ele realizando as atividades com a criança. Caso o cuidador tivesse dúvidas ao longo da semana, ele poderia enviar perguntas pelo grupo criado no aplicativo de mensagens, e o profissional respondia por escrito o mais breve possível; 9) O cuidador deveria enviar no grupo do aplicativo de mensagens, um dia antes da sessão por videoconferência, as fotos dos protocolos preenchidos e os vídeos realizados ao longo da semana; 10) Antes da nova sessão com o cuidador, o profissional assistia aos vídeos e analisava os protocolos: a partir desses materiais, ele avaliava quais ajustes precisava orientar o cuidador a fazer, quais atividades novas poderiam ser implementadas e quais atividades já haviam atingido critérios de aprendizagem e/ou manutenção. Sempre que necessário, o profissional discutia algum ponto ou tirava alguma dúvida com o supervisor. É importante ressaltarmos que semanalmente o profissional tinha pelo menos um horário (50 minutos) com o supervisor, para discutir as intervençóes realizadas com cada criança que ele acompanhava, por isso o supervisor também estava presente no grupo criado no aplicativo de mensagens; 11) A partir da análise e decisão do profissional, protocolos de atividades novas eram enviados para o responsável pela criança; 12) Uma nova sessão realizada por videoconferência ocorria, e o processo descrito nos passos anteriores se repetia. 


\subsection{CONSIDERAÇÓES ÉTICAS E FIDEDIGNIDADE}

A coleta dos dados foi realizada por meio das avaliaçóes aplicadas pelos profissionais do CEI e dos protocolos de registro das atividades, preenchidos pelos cuidadores (que se referiam às atividades que eles realizavam com as crianças). A coleta e a análise dos dados foram realizadas entre janeiro de 2018 e maio de 2019.

Os procedimentos empregados neste estudo foram aprovados pelo Comitê de Ética da Faculdade de Ciências Médicas de Minas Gerais para um projeto maior que pretendia avaliar os efeitos de Intervençóes Comportamentais Intensivas realizadas por meio da capacitação de cuidadores.

Dois avaliadores independentes contabilizaram 30\% das avaliaçóes (realizadas pelos profissionais do CEI). Fez-se o cálculo de concordância entre observadores, usando-se a fórmula: concordâncias divididas pela somatória de concordâncias e discordâncias, multiplicado por cem (Kazdin, 1982). O coeficiente de concordância para as avaliaçóes foi de 100\%.

\section{Resultados}

Os cuidadores eram estagiários de Psicologia (P1, P3, P5, P7, P9, P10, P11, P13, P14, P15, P16, P19, P22, P23), mães (P8, P12, P17, P20), pais (P2, P18, P21) e babás (P4, P6, P24); 79\% tinha concluído o nível médio de escolaridade e o restante tinha o nível superior. Esses cuidadores foram capazes de realizar com as crianças com autismo de 9 a 41 programas de ensino (Tabela 1). Os efeitos da capacitaçáo dos cuidadores, implementada com o uso de TICs, foram medidos por meio do desempenho das crianças com autismo nas avaliaçóes, aplicadas no início e no término da intervenção.

\section{Tabela 1}

Características gerais dos participantes

\begin{tabular}{|c|c|c|c|c|c|c|c|c|c|c|}
\hline \multirow{2}{*}{ P. } & \multirow{2}{*}{$\begin{array}{l}\text { Gêne- } \\
\text { ro }\end{array}$} & \multicolumn{2}{|c|}{ Idade } & \multicolumn{2}{|c|}{ Fala } & \multirow{2}{*}{$\begin{array}{c}\text { Intervalo } \\
\text { (meses) } \\
\text { A2-A1 }\end{array}$} & \multirow{2}{*}{$\begin{array}{c}\text { Progra- } \\
\text { mas }\end{array}$} & \multirow{2}{*}{$\begin{array}{c}\text { Distân- } \\
\text { cia } \\
(\mathbf{k m})\end{array}$} & \multirow{2}{*}{ Cuidador } & \multirow{2}{*}{ Escolaridade } \\
\hline & & A1 & A2 & A1 & A2 & & & & & \\
\hline P1 & $\mathrm{F}$ & $3 \mathrm{a} 2 \mathrm{~m}$ & $4 \mathrm{a} 2 \mathrm{~m}$ & não & $\operatorname{sim}$ & 12 & 33 & 441 & estagiária & médio \\
\hline P2 & M & $3 \mathrm{a} 2 \mathrm{~m}$ & $4 \mathrm{a} 2 \mathrm{~m}$ & não & $\operatorname{sim}$ & 12 & 20 & 885 & pais & médio \\
\hline P3 & F & $3 \mathrm{a} 4 \mathrm{~m}$ & $4 a 7 m$ & não & $\operatorname{sim}$ & 17 & 35 & 441 & estagiário & médio \\
\hline P4 & $\mathrm{F}$ & $3 \mathrm{a} 5 \mathrm{~m}$ & $4 \mathrm{a} 4 \mathrm{~m}$ & $\operatorname{sim}$ & $\operatorname{sim}$ & 11 & 20 & 12,4 & babá & médio \\
\hline P5 & M & $3 \mathrm{a} 10 \mathrm{~m}$ & $4 \mathrm{a} 7 \mathrm{~m}$ & $\operatorname{sim}$ & $\operatorname{sim}$ & 9 & 16 & 2.367 & estagiária & médio \\
\hline P6 & M & $3 \mathrm{a} 10 \mathrm{~m}$ & $5 a$ & $\operatorname{sim}$ & $\operatorname{sim}$ & 14 & 24 & 584 & babá & superior \\
\hline P7 & M & $4 \mathrm{a} 1 \mathrm{~m}$ & $4 \mathrm{a} 10 \mathrm{~m}$ & nấo & $\operatorname{sim}$ & 10 & 22 & 2.367 & estagiária & médio \\
\hline P8 & M & $4 \mathrm{a} 1 \mathrm{~m}$ & $5 \mathrm{a} 1 \mathrm{~m}$ & não & $\operatorname{sim}$ & 12 & 21 & 53,1 & mãe & médio \\
\hline P9 & M & $4 \mathrm{a} 9 \mathrm{~m}$ & $5 \mathrm{a} 9 \mathrm{~m}$ & $\operatorname{sim}$ & $\operatorname{sim}$ & 12 & 41 & 340 & estagiária & médio \\
\hline P10 & M & $4 \mathrm{a} 10 \mathrm{~m}$ & $5 \mathrm{a} 9 \mathrm{~m}$ & nẫo & $\operatorname{sim}$ & 11 & 39 & 449 & estagiária & médio \\
\hline P11 & M & $5 \mathrm{a} 1 \mathrm{~m}$ & $6 \mathrm{a} 7 \mathrm{~m}$ & não & $\operatorname{sim}$ & 18 & 38 & 340 & estagiária & médio \\
\hline P12 & M & $5 \mathrm{a} 3 \mathrm{~m}$ & $6 \mathrm{a} 5 \mathrm{~m}$ & nẫo & não & 15 & 14 & 1.228 & mãe & médio \\
\hline P13 & M & $5 \mathrm{a} 3 \mathrm{~m}$ & $6 \mathrm{a} 2 \mathrm{~m}$ & não & $\operatorname{sim}$ & 11 & 34 & 423 & estagiária & superior \\
\hline
\end{tabular}




\begin{tabular}{|c|c|c|c|c|c|c|c|c|c|c|}
\hline P14 & M & $5 \mathrm{a} 3 \mathrm{~m}$ & $6 \mathrm{a} 3 \mathrm{~m}$ & não & não & 12 & 34 & 340 & estagiária & médio \\
\hline P15 & M & $5 \mathrm{a} 4 \mathrm{~m}$ & $6 \mathrm{a} 5 \mathrm{~m}$ & não & $\operatorname{sim}$ & 13 & 34 & 340 & estagiária & médio \\
\hline P16 & M & $5 \mathrm{a} 8 \mathrm{~m}$ & $6 \mathrm{a} 11 \mathrm{~m}$ & nấo & nấo & 15 & 14 & 2.367 & estagiária & médio \\
\hline P17 & M & $6 a$ & $7 \mathrm{a} 1 \mathrm{~m}$ & não & $\operatorname{sim}$ & 13 & 14 & 156 & mãe & superior \\
\hline P18 & M & $6 a$ & $7 \mathrm{a} 3 \mathrm{~m}$ & não & não & 15 & 16 & 536 & pais & superior \\
\hline P19 & M & $6 \mathrm{a} 3 \mathrm{~m}$ & $7 \mathrm{a} 4 \mathrm{~m}$ & $\operatorname{sim}$ & $\operatorname{sim}$ & 13 & 25 & 340 & estagiária & médio \\
\hline P20 & $\mathrm{F}$ & $6 \mathrm{a} 5 \mathrm{~m}$ & $7 \mathrm{a} 6 \mathrm{~m}$ & $\operatorname{sim}$ & $\operatorname{sim}$ & 13 & 14 & 653 & mãe & médio \\
\hline P21 & M & $6 \mathrm{a} 9 \mathrm{~m}$ & $7 \mathrm{a} 9 \mathrm{~m}$ & não & $\operatorname{sim}$ & 12 & 9 & 39,3 & pais & médio \\
\hline P22 & M & $8 a$ & $9 \mathrm{a} 4 \mathrm{~m}$ & não & $\operatorname{sim}$ & 16 & 20 & 584 & estagiária & superior \\
\hline P23 & M & $8 \mathrm{a} 1 \mathrm{~m}$ & $9 \mathrm{a} 7 \mathrm{~m}$ & não & nấo & 18 & 41 & 423 & estagiária & médio \\
\hline \multirow[t]{2}{*}{ P24 } & M & $8 \mathrm{a} 10 \mathrm{~m}$ & $10 \mathrm{a} 3 \mathrm{~m}$ & não & $\operatorname{sim}$ & 17 & 13 & 97,1 & babá & médio \\
\hline & $\begin{array}{l}\text { Mé- } \\
\text { dia }\end{array}$ & $5 \mathrm{a} 3 \mathrm{~m}$ & $6 \mathrm{a} 4 \mathrm{~m}$ & $25 \%$ & $79,1 \%$ & 13,3 & 24,6 & 658,5 & & \\
\hline
\end{tabular}

Nota. Participante com Autismo, gênero, idade, presença de fala, intervalo em meses entre as avaliaçôes, número de programas de ensino; distância da sede do CEI; cuidador e escolaridade do cuidador. Dados da pesquisa.

A Figura 1 apresenta a distribuição dos dados considerando os resultados das crianças na CARS, PEP-R e IPO em pré (Avaliação 1: A1) e pós testes (Avaliação 2: A2). As pontuações médias final e inicial da CARS indicaram diminuição dos sintomas de autismo de 37,6 (autismo grave) para 33 (autismo leve/moderado); a pontuação média no PEP-R indicou ganho no desenvolvimento (pontuação de 56,5 para 78,7) e no IPO também (pontuação de 174,3 para 257,9). Um tratamento estatístico foi realizado por meio do teste de Wilcoxon (Field, 2009) para amostras pareadas com o objetivo de verificar se havia diferenças significativas no desempenho dos participantes em pré e pós-testes. As diferenças foram consideradas significativas no nível de probabilidade menor do que 0,05 ( $<<0,05)$; acima desse valor, a hipótese nula de que as médias eram idênticas não seria rejeitada. Os resultados em todas as análises (CARS: 0,001; PEP-R: 0,001; IPO: 0,005) indicaram valores de p menores do que 0,05 - valores estatisticamente significativos.

\section{Figura 1}

Distribuição dos dados considerando os resultados da CARS, PEP-R e IPO

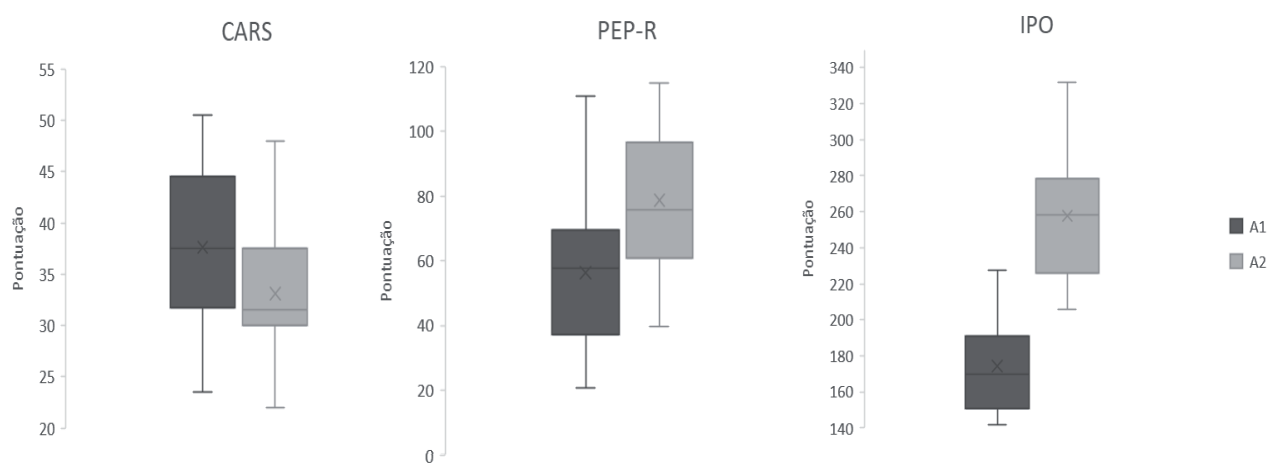


A Figura 2 apresenta as pontuações médias dos participantes em pré e pós testes, por áreas do desenvolvimento no PEP-R e no IPO; houve aumento da pontuação média em todas as áreas do desenvolvimento avaliadas. Tratamento estatístico também foi realizado por meio do teste de Wilcoxon para amostras pareadas com o objetivo de verificar se havia diferenças significativas no desempenho dos participantes por áreas do desenvolvimento no PEP-R e no IPO; observou-se diferenças estatisticamente significativas em todas as áreas (ver Tabela 2).

\section{Figura 2}

Pontuaçóes médias dos participantes em pré (a1) e pós (a2) testes por áreas do desenvolvimento no PEP-R e no IPO

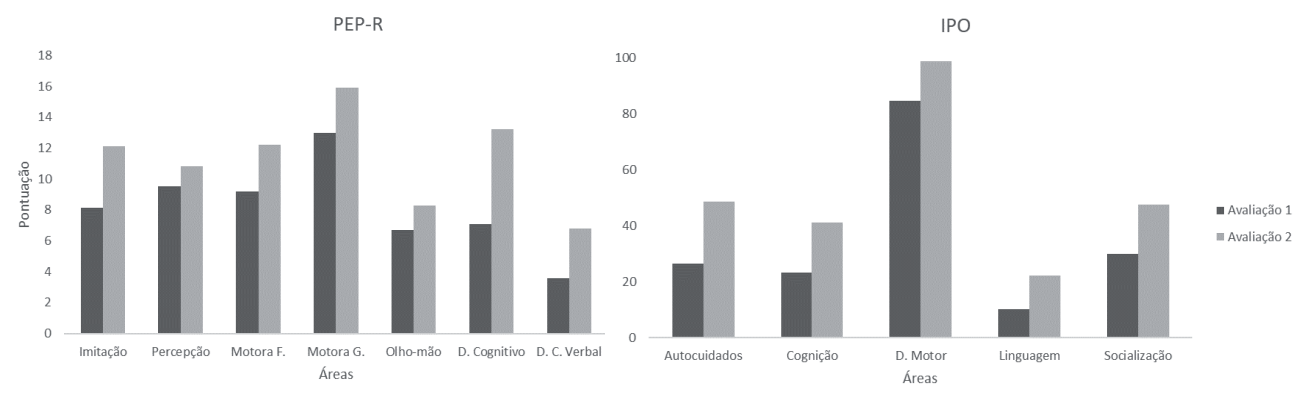

Tabela 2

Médias e p-valor dos participantes com Autismo no PEP-R e no IPO por área do desenvolvimento

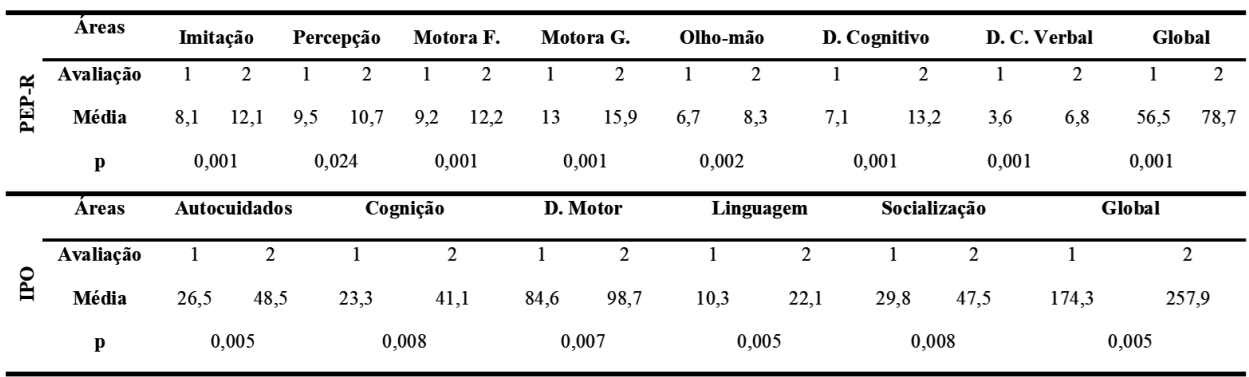

\section{Discussão E CONCLUSÓES}

A complexidade e a gravidade dos sintomas do autismo podem comprometer a qualidade de vida das pessoas afetadas e de outras que convivem com elas. Atualmente, há dezenas de tratamentos direcionados à essa população, porém nem todos apresentam evidências científicas suficientes que possam indicar efetividade. Até o momento, os tratamentos que apresentaram os melhores resultados são aqueles realizados de maneira intensiva (muitas horas semanais de estimulação) e fundamentados em análise do comportamento (Medavarapu et al., 2019), definidos como Intervenção Comportamental Intensiva. Há muitos modelos de intervençóes 
comportamentais e intensivas destinados ao tratamento do autismo e, apesar das diferenças entre eles, esses modelos têm em comum a realização de estimulações amplas, que afetam várias áreas do desenvolvimento simultaneamente, empreendidas predominantemente de maneira individualizada e por muitas horas semanais (Romanczyk \& McEachin, 2016).

Apesar da ampla evidência de efetividade dos modelos de Intervenção Comportamental Intensiva, o acesso a esse tipo de tratamento ainda é escasso; há alta demanda de pessoas com autismo, poucos profissionais capacitados e custo elevado, em função das muitas horas semanais de terapia que são necessárias. Nesse sentido, a capacitação de pais e cuidadores tem sido empregada em diversos estudos, que indicaram a viabilidade e a efetividade dessa prática (Anderson et al., 1987; Dawson et al., 2010; McConachie \& Diggle, 2007, Sheinkopf \& Sielgel, 1998; Sherman et al., 1988; Smith et al., 2000).

Três estudos nacionais apresentaram dados a respeito da capacitação de cuidadores para a realização de Intervenção Comportamental Intensiva. Gomes et al. (2017) avaliaram os efeitos do primeiro ano de Intervenção Comportamental Intensiva no desenvolvimento de nove crianças com autismo, com idades entre 1 ano e 3 meses e 2 anos e 11 meses, atendidas por um centro brasileiro especializado. A intervençáo, conduzida por aproximadamente 15 horas semanais, foi realizada na residência dos participantes por meio da capacitação dos cuidadores. As crianças foram avaliadas no início e ao término da intervenção. Os dados foram analisados individualmente e os resultados indicaram ganhos no desenvolvimento de todas as crianças. Posteriormente, Gomes et al. (2019), ao utilizarem um delineamento de grupo, avaliaram os efeitos desse tipo de intervenção, realizada por meio da capacitação de cuidadores, no desenvolvimento de crianças com autismo. As autoras também compararam as crianças que realizaram a Intervenção Comportamental Intensiva com crianças que não realizaram. Os resultados indicaram ganhos significativos em todas as áreas do desenvolvimento das crianças com autismo que passaram pela intervenção intensiva, enquanto as crianças do outro grupo apresentaram ganhos menos expressivos.

Na sequência, Andalécio et al. (2019) apresentaram um estudo de caso único com uma criança com autismo gravemente comprometida e não falante. A intervenção ocorreu por 40 horas semanais em ambiente domiciliar e escolar, por meio da capacitação dos cuidadores, ao longo de cinco anos consecutivos. Instrumentos padronizados foram utilizados para medir o desenvolvimento da criança. Os resultados gerais indicaram ganhos no desenvolvimento da criança e a viabilidade da capacitação dos cuidadores para esse tipo de intervençáo.

O estudo atual também pretendeu avaliar os efeitos da capacitação de cuidadores de pessoas com autismo para a realização de Intervenção Comportamental Intensiva, porém se difere dos estudos anteriores por avaliar os efeitos da intervenção realizada por cuidadores que foram capacitados por meio do uso de TICs, estando profissionais e cuidadores em locais diferentes e sem contato direto; nos estudos anteriores os cuidadores foram capacitados em contato direto com os profissionais especializados.

Os resultados deste estudo são condizentes com os da literatura ao indicar ganhos no desenvolvimento das crianças com autismo, após realizarem Intervenção Comportamental Intensiva, mesmo quando essa intervenção não é implementada por profissionais especializados, mas executada por meio de cuidadores capacitados por profissionais especializados 
(Andalécio et al., 2019; Anderson et al., 1987; Dawson et al., 2010; Gomes et al., 2017; Gomes et al., 2019; McConachie \& Diggle, 2007, Sheinkopf \& Sielgel, 1998; Sherman et al., 1988; Smith et al., 2000).

Os ganhos no desenvolvimento dos participantes com autismo provavelmente podem ser atribuídos à intervenção intensiva realizada pelos cuidadores, pois, além dessa intervenção, as crianças realizavam apenas intervenção não intensiva (sessóes de fonoaudiologia e/ ou terapia ocupacional). Nesse sentido, a literatura indica com bastante consistência que intervenções não intensivas não são suficientes para promover efeitos robustos e em várias áreas do desenvolvimento de pessoas com autismo (Lovaas, 1987; Sherman et al., 1988; Smith, 1999; Warren et al., 2011). Outra possibilidade é considerar que o efeito no desenvolvimento pode ter sido decorrente da maturação das crianças, visto que o passar dos meses pode ter provocado ganhos no desenvolvimento das crianças. Contudo, isso é pouco provável, pois a literatura indica que o autismo é crônico e que não é comum a melhora "espontaneamente" sem intervenção intensiva e adequada (APA, 2013; Gomes et al., 2019).

Além dos ganhos observados nas crianças participantes deste estudo, que provavelmente são decorrentes das atividades que os cuidadores realizaram com elas, os resultados também indicaram a viabilidade do uso de TICs para a capacitação dos cuidadores, em um contexto de Intervenção Comportamental Intensiva, pois os cuidadores foram capazes de realizar as atividades, sob orientação de profissionais especializados, estando profissionais e cuidadores em locais diferentes.

Os resultados deste estudo são animadores ao indicar a viabilidade da capacitação de cuidadores de crianças com autismo por meio do uso de TICs e efeitos importantes dessa capacitaçáo no desenvolvimento das crianças com autismo. Entretanto, novos estudos devem dar continuidade à exploração das possibilidades aqui demonstradas, com controle metodológico mais rigoroso (para além do delineamento de pré e pós-testes) e que forneçam outras medidas do desempenho das crianças e dos cuidadores. Adicionalmente, encoraja-se a comparação dos efeitos de intervençóes realizadas presencialmente com intervençóes realizadas por meio do uso de TICs.

\section{REFERÊNCIAS}

American Psychiatry Association (2002). Manual diagnóstico e estatístico de transtornos mentais- texto revisado. Artes Médicas.

American Psychiatry Association (2013). Diagnostic and statistical manual of mental disorders - DSM-5 ( $5^{\text {th }}$ ed). American Psychiatric Association.

Andalécio, A., Gomes, C., Silveira, A., Oliveira, I., \& Castro, R. (2019). Efeitos de 5 anos de intervenção comportamental intensiva no desenvolvimento de uma criança com autismo. Revista Brasileira de Educação Especial, 25(3), 389-402. https://doi.org/10.1590/s1413-65382519000300003

Anderson, S. R., Avery, D. L., DiPietro, E. K., Edwards, G. L., \& Christian, W. P. (1987). Intensive home-based early intervention with autistic children. Education and Treatment of Children, 10(4), 352-366.

Associação Brasileira de Psicologia e Medicina Comportamental (2019). Acreditados. Acreditação. http:// acreditacao.abpmc.org.br/acreditados.php 
Baer, D. M., Wolf, M. M., \& Risley, T. R. (1987). Some still-current dimensions of applied behavior analysis. Journal of Applied Behavior Analysis, 20(4), 91-97.

Baio, J., Wiggins, L., Christensen, D. L., Maenner, M. J., Daniels, J., Warren, Z., \& Durkin, M. S. (2018). Prevalence of autism spectrum disorder among children aged 8 years-autism and developmental disabilities monitoring network, 11 sites, United States, 2014. MMWR Surveillance Summaries, 67(6), 1.-23

Barboza, A. A., Silva, A. J. M., Barros, R. S., \& Higbee, T. S. (2015). Efeitos de videomodelação instructional sobre o desempenho de cuidadores na aplicaçáo de programas de ensino a crianças diagnosticadas com autismo. Acta Comportamentalia, 23(4), 405-421.

Barboza, A. A., Costa, L. C. B., \& Barros, R. D. S. (2019). Instructional videomodeling to teach mothers of children with autism to implement discrete trials: a systematic replication. Trends in Psychology, 27(3), 795-804. http://doi.org/10.9788/tp2019.3-14

Boisvert, M., Lang, R., Andrianopoulos, M., \& Boscardin, M. L. (2010). Telepractice in the assessment and treatment of individuals with autism spectrum disorders: A systematic review. Developmental Neurorehabilitation, 13(6), 423-432. https://doi.org/10.3109/17518423.2010.499889

Conselho Federal de Psicologia (2019). A psicologia brasileira apresentada em números. CFP. http:// www2.cfp.org.br/infografico/quantos-somos/

Dawson, G., Rogers, S., Munson, J., Smith, M., Winter, J., Greenson, J., Donaldson, A., \& Varley, J. (2010). Randomized, controlled trial of an intervention for toddlers with autism: The Early Start Denver Model. Pediatrics, 125(1), 17-23. https://doi.org/10.1542/peds.2009-0958

Field, A. (2009). Descobrindo a estatística usando o SPSS-2. Bookman.

Gomes, C. G. S. (2015). Ensino de leitura para pessoas com autismo. Appris.

Gomes, C. G. S., \& Silveira, A. D. (2016). Ensino de habilidades básicas para pessoas com autismo: Manual para Intervenção Comportamental Intensiva. Appris.

Gomes, C. G. S., De Souza, D. das G., Silveira, A. D., \& Oliveira, I. M. (2017). Intervenção comportamental precoce e intensiva com crianças com Autismo por meio da capacitação de cuidadores. Revista Brasileira de Educação Especial, 23(3), 377-390. https://doi.org/10.1590/s141365382317000300005

Gomes, C. G. S., De Souza, D. das G., Silveira, A. D., Rates, A. C., Paiva, G. C. de C., \& Castro, N. P. de. (2019). Efeitos da Intervenção Comportamental Intensiva realizada por meio da capacitação de cuidadores de crianças com autismo. Psicologia: Teoria e Pesquisa, 35, e3523. https://doi. org/10.1590/0102.3772e3523

Instituto Brasileiro de Geografia e Estatística (2019). Projeção da População do Brasil e das Unidades da Federação. Recuperado em 20 de agosto de 2020 de https:/www.ibge.gov.br/apps/populacao/ projecao/index.html?utm_source=portal\&utm_medium=popclock\&utm_campaign=novo_ popclock

Kazdin A. E. (1982). Single-case research designs: Methods for clinical and applied settings. Oxford University Press.

Leon, V. C., Bosa, C. A., Hugo, C. N., \& Hutz, C. (2004). Propriedades psicométricas do Perfil Psicoeducacional Revisado: PEP-R. Avaliação Psicológica, 3(1), 39-52.

Lovaas, O. I. (1981). The ME book. Pro-Ed. 
Lovaas, O. I. (1987). Behavioral treatment and normal educational and intellectual functioning in young autistic children. Journal of Consulting and Clinical Psychology, 55(1), 3-9.

McConachie, H., \& Diggle, T. (2007). Parent implemented early intervention for young children with autism spectrum disorder: A systematic review. Journal of Evaluation in Clinical Practice, 13(1), 120-129. https://doi.org/10.1111/j.1365-2753.2006.00674.x

Medavarapu, S., Marella, L. L., Sangem, A., \& Kairam, R. (2019). Where is the evidence? A narrative literature review of the treatment modalities for Autism Spectrum Disorders. Cureus, 11(1), e3901. https://doi.org/10.7759/cureus.3901

Organização Mundial de Saúde (1993). Classificação de transtornos mentais e de comportamentos da CID10: descriçôes clínicas e diretrizes diagnósticas. Artmed.

Pereira, A., Riesgo, R. S., \& Wagner, M. B. (2008). Autismo infantil: tradução e validação da Childhood Autism Rating Scale para uso no Brasil. Jornal de Pediatria, 84(6), 487-494.

Romanczyk, R. G., \& McEachin, J. (Eds.). (2016). Comprehensive models of autism spectrum disorder treatment: Points of divergence and convergence. Springer.

Schopler, E., Reichler, J. R., \& Renner, C. (1988). CARS-The Childhood Autism Rating Scale. Westerm Pychological Services.

Schopler, E., Reichler, R. J., Bashford, A., Lansing, M. D., \& Marcus, L. M. (1990). Individualized assessment and treatment for autistic and developmentally disabled children: Psychoeducational ProfileRevised (PEP-R). Pro- Ed.

Sheinkopf, S. J., \& Siegel, B. (1998). Home-based behavioral treatment of young children with autism. Journal of Autism and Developmental Disorders, 28(1), 15-23.

Sherman, J., Barker, P., Lorimer, P., Swinson, R., \& Factor, D. C. (1988). Treatment of autistic children: Relative effectiveness of residential, out-patient and home-based interventions. Child Psychiatry and Human Development, 19(2), 109-125.

Silveira, A. D., \& Gomes, C. G. (2019). Ensino de habilidades de autocuidados para pessoas com autismo: Manual para Intervenção Comportamental Intensiva. CEI.

Smith, T., Buch, G. A., \& Gamby, T. E. (2000). Parent-directed, intensive early intervention for children with pervasive developmental disorder. Research in Developmental Disabilities, 21(4), 297-309.

Smith, T. (1999). Outcome of early intervention for children with autism. Clinical Psychology: Science and Practice, 6(1), 33-49.

Vismara, L. A., Young, G. S., Stahmer, A. C., Griffith, E. M., \& Rogers, S. J. (2009). Dissemination of evidence-based practice: Can we train therapists from a distance? Journal of autism and developmental disorders, 39(12), 1636.

Warren, Z., McPheeters, M. L., Sathe, N., Foss-Feig, J. H., Glasser, A., \& Veenstra-VanderWeele, J. (2011). A systematic review of early intensive intervention for autism spectrum disorders. Pediatrics, 127(5), 1303-1311.

Willians, L. A., \& Aiello, A. L. R. (2001). Inventário Portage operacionalizado. Mennon.

Recebido em: 01/05/2020

Reformulado em: 04/08/2020

Aprovado em: 17/10/2020 
GOMES, C.G.S. et al. 